\title{
Design and characterization of a portable ultrasonic indoor 3D positioning system
}

\author{
Alessio De Angelis, Antonio Moschitta, Paolo Carbone \\ Dept. of Engineering \\ University of Perugia \\ Via G. Duranti, 93 - 06125 Perugia - Italy \\ \{alessio.deangelis, antonio.moschitta, paolo.carbone\}@unipg.it
}

\author{
Massimo Calderini, Stefano Neri, Renato Borgna, \\ Manuelo Peppucci \\ Società delle Fucine S.r.L. \\ Viale B. Brin, 218 - 05100 Terni, Italy \\ \{massimo.calderini, stefano.neri, \\ quality.control.sdf, M.peppucci\}@acciaiterni.it
}

\begin{abstract}
In this paper, an ultrasonic positioning system is presented and characterized, based on the usage of a portable grid of beacons and of a few fixed anchors. Since the beacon grid can be moved to guarantee line of sight transmissions, the proposed strategy is potentially suitable for accurate positioning of a mobile object in an environment with a complex geometry. The system was tested experimentally, exhibiting a subcentimeter positioning accuracy in a range up to $4 \mathrm{~m}$.
\end{abstract}

Keywords-Ultrasound indoor positioning; portable; mobile; chirp; fitting

\section{INTRODUCTION}

Indoor positioning is an interesting topic and an enabler in several fields of application, and consequently subject to a significant number of research activities. Various techniques have been proposed in recent years, mostly based on preliminary ranging between a mobile node and a set of fixed beacons, whose position is known in advance, followed by a triangulation algorithm [1]. Range measurements are mostly obtained by transmitting a signal between each beacon and the mobile node, by measuring one or more parameters in the received signal, and by inverting a suitable propagation model, relating the parameters to the distance. Typically measured parameters are Received Signal Strength (RSS), Angle of Arrival $(A o A)$, and Time of Flight $(T o F)$. Several signaling schemes have been proposed, based on the transmission of Ultra Wide Band (UWB) RF signals [2-6], narrowband RF signals [7-10], inductively coupled low frequency EM signals [11-15], optical signals, both for ranging and for image reconstruction based positioning [16-17], and ultrasonic acoustic signals [18-24]. The considered approaches may be competitive depending on the scenario requirements, expressed in terms of accuracy and measurement rate, Line of Sight (LoS) availability, power consumption, and processing power [1].

In this paper, a system based on ultrasound transmissions is considered. Ultrasonic systems are an attractive solution in indoor applications, since they are low power, and provide a good accuracy in LoS condition. Low cost transceivers are also available, and the involved frequencies allow avoiding expensive hardware or exceedingly complex processing. Such features attracted research interests, and recent activities on this topic are mentioned in the literature [18]-[24]. For instance, in [24] a sub-centimeter accurate positioning system is presented, based on a set of 4 ultrasound emitting beacons. In [24] the fixed beacons, mounted on the ceiling of a room in a square configuration, are triggered via radio signals. They transmit ultrasound pulses, obtained as binary phase shift keying (BPSK) modulated waveforms about a center frequency of 40 $\mathrm{kHz}$, with a bandwidth of about $2 \mathrm{kHz}$. Bandpass sampling is then exploited to relax processing requirements. $T o F$ is estimated as the delay between the triggering RF pulse and the envelope maximum in the received ultrasound pulses.

In this paper, a different positioning system based on ultrasound signaling is presented. As in [24] ToF measurements between a mobile node and a set of beacons are collected, converted in range measurements, and used to triangulate the position of a mobile node. The presented system is designed to work in large industrial buildings, with a ceiling several meters high, exceeding the range of solutions like [21][24]. Moreover, industrial applications may require to locate a mobile node moving on an irregular geometry (i.e., the curved surface of some artifact), so that a fixed grid of beacons may not guarantee continuity of service. In fact, depending on the artifact size and shape and on the presence of large machinery in the operational environment, LoS transmissions between the mobile node and the beacons may not always be possible, and multipath effects may alter the received signals.

To circumvent these problems, the presented system measures the relative position of the mobile node with respect to a portable beacon grid, realized by a set of ultrasound transceivers mounted on a planar panel. The beacon grid can locate itself accurately by means of ToF measurements, with respect to a small set of known positions ("anchors"), located in such a way that LoS is always guaranteed between such positions and the beacons. Thus, the mobile node position, once measured with respect to the beacon grid, can be converted in a different set of coordinates, referred to a fixed position in the experimental environment. If the mobile node 


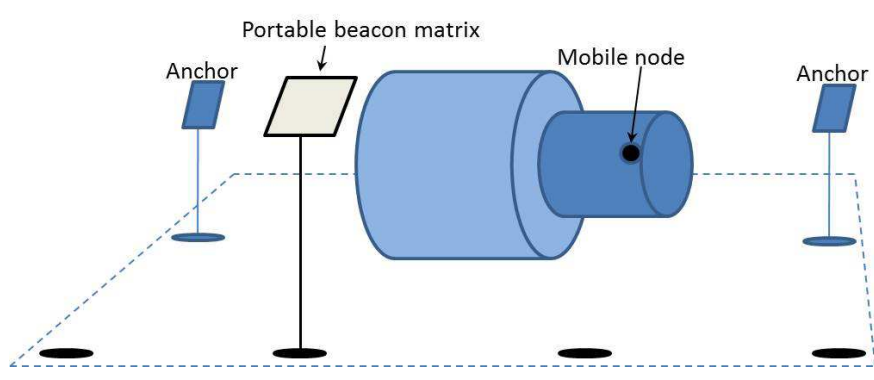

Fig. 1: layout of the proposed ultrasonic positioning system

explores a set of positions that do not allow LoS transmissions with the beacon grid, the beacon grid itself can be moved to a more suitable location. Then, the beacon grid can recalibrate its own position by measuring the position of the fixed anchors, and new measurements of the mobile node position can again be referred to the fixed reference. Notice that the calibration may be performed by placing additional ultrasound transceivers in the anchors' positions, or by placing there the mobile node during the calibration phase.

Thus, with respect to systems like [24]-[25], the usage of a portable beacon grid provides location estimation also in non LoS conditions, is flexible in reducing the distance between the mobile node and the beacons, and can reduce installation costs.

Another difference with respect to [24] is that, in order to achieve good accuracy, linear chirp signals are used as transmitted ultrasound pulses [25]. This solution is potentially more robust to multipath, since the receiver can use correlation techniques to discriminate the ultrasound pulse received through the LoS path from multipath induced replicas, even if the received pulses overlap. To the authors' knowledge, such an ultrasound based positioning system has not been realized and tested yet. It is worth noting that a similar solution has been recently presented on the market [25], that uses RFID signals to trigger ultrasound transmissions and a 4 beacon portable grid. With respect to [25], the proposed system is based on dynamic recalibration to support relocations of the portable beacon grid, a redundant beacon panel is adopted, using a larger number of beacons to improve accuracy, and design criteria to meet targeted accuracy are given, with respect to grid size, number of beacons, and ultrasound signal's parameters.

The proposed system was developed over several steps. At first, an accuracy analysis was carried out, relating the achievable accuracy to the beacon panel layout, air temperature, and $T o F$ measurement accuracy. This system was progressively refined, and validated experimentally. The proposed solution was initially validated in a ranging context, identifying the main contributors to measurement uncertainty. Then, by extending the results of [26], a robust ranging procedure was developed, guaranteeing sub-centimeter accuracy, and a 3D position estimation.

\section{SYSTEM ARCHITECTURE AND SIGNALLING SCHEME}

\section{A. System architecture and measurement procedure}

The system layout is shown in Fig. 1, that shows the portable beacon grid, the mobile node, the irregular surface, and the fixed anchors. The mobile grid can be hand placed in a set of nominal positions and orientations with respect to an absolute reference by an operator. Consequently, depending on the size and shape of the artifact, different positions assumed by the mobile node can always be estimated in LoS conditions. Then, ToF measurements between the transceivers in the beacon grid and the mobile nodes are carried out. The results are fed to a triangulation algorithm, based on numerical fitting, that provides relative position of the mobile node with respect to the beacon grid. The numerical fitting minimizes the cost function

$$
\begin{aligned}
& F=\left[F_{1}, \ldots F_{N_{b}}\right], \\
& F_{i}=v \cdot t_{i}-d_{i}(\hat{x}, \hat{y}, \hat{z}), \quad i=1, \ldots, N_{b}
\end{aligned}
$$

where $N_{b}$ is the number of beacons, $(\hat{x}, \hat{y}, \hat{z})$ is the vector of estimators of the coordinates $(x, y, z)$ of the mobile node, $v$ is the speed of sound in the propagation medium, is the ToF between the mobile node and the i-th beacon, and $d_{i}(\hat{x}, \hat{y}, \hat{z})$ is the Euclidean distance between the $i$-th beacon and the estimated mobile node position. If the position of the beacon grid were perfectly known, the relative coordinates could be transformed in the absolute coordinates straightforwardly. However, since the placement of the beacon grid may be inaccurate, the corresponding positioning error also affects the mobile node estimated position, when translated in the absolute reference. Thus, a preliminary calibration procedure was considered. It begins with the measurement of the relative position of the fixed anchors with respect to the beacon grid. In fact, a placement error of the beacon grid can be considered as a rigid roto-translation. Since the anchors are fixed, their positions are known in advance in the absolute reference, with very good accuracy. Consequently, the difference between the coordinates of the absolute reference and the measured ones can be obtained, and a linear transformation $R$ can be identified, describing the rigid linear transformation that maps the estimated positions of the anchors into the true ones. Hence, when the mobile node coordinates $(x, y, z)$ are estimated, a refined position estimate $\left(x_{c}, y_{c}, z_{c}\right)$ can be obtained as

$$
\left(x_{c}, y_{c}, z_{c}\right)=R(\hat{x}, \hat{y}, \hat{z})=R_{A} \cdot(\hat{x}, \hat{y}, \hat{z})^{T}+R_{B},
$$

where $R_{A}$ is a $3 \times 3$ matrix and $R_{B}$ is a $3 \times 1$ vector, for a total of 12 parameters. The proposed solution trades versatility (guaranteed LoS) for a reduction in accuracy, due to the additional uncertainty source introduced by the portable beacon grid positioning.

\section{B. Transmitted signals and propagation model}

Since the system is ToF based, a linear chirp pulse $s(t)$ with constant envelope $A$ was selected as transmitted ultrasonic signal, defined as

$$
\begin{aligned}
& s(t)= \begin{cases}A \sin (2 \pi f(t) \cdot t), & 0 \leq t \leq t_{d} \\
0, & \text { elsewhere }\end{cases} \\
& f(t)=f_{\text {low }}+\frac{f_{\text {high }}-f_{\text {low }}}{t_{d}} t, \quad 0 \leq t \leq t_{d}
\end{aligned}
$$

such that the sinewave frequency spans the $\left[f_{\text {low }}, f_{\text {high }}\right]$ interval in a time $t_{d}$ [25]. Chirp signaling is a well-known technique, 

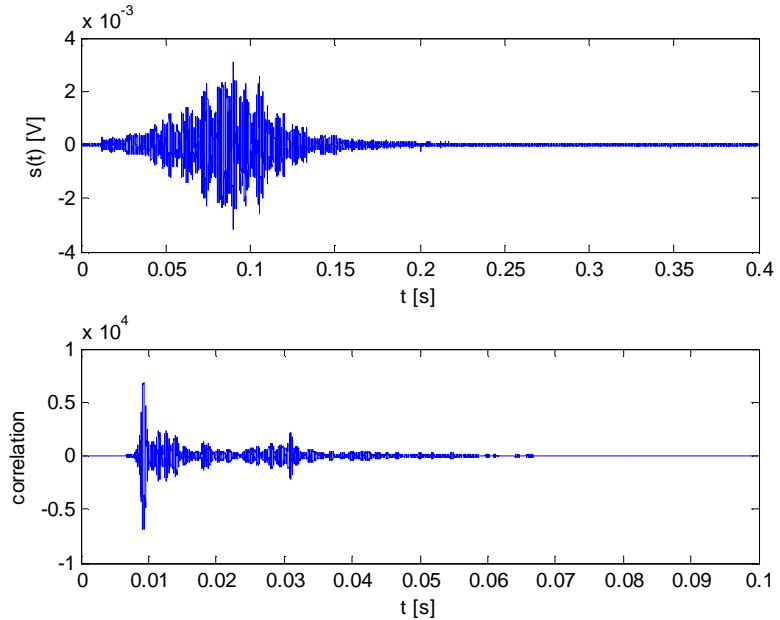

Fig. 2:experimental results for a chirp transmission affected by multipath. The upper plot shows a received signal, comprising a

LoS contribution partially overlapping with some multipath

replicas, while the lower plot shows the correlation of the received signal with a locally stored template of the chirp pulse.

that unlike threshold detectors, allows the resolution of multipath components even if the useful signal and the replicas overlap in the time domain. In particular, the signal $T o F$ is estimated by evaluating peaks of a discrete sequence, obtained by correlating the received signal and a template, assumed to be synchronized to the transmission time of the ultrasound pulse. The synchronization may be achieved either by transmitting a copy of the pulse via an electric channel, or by storing a sampled replica in the receiver, and transmitting a trigger pulse on the electric channel. This approach provides a good temporal resolution and discrimination properties, because a signal composed of multiple overlapping replicas of the transmitted pulse simply results in multiple peaks in the correlation sequence, one per replica. Coupled with the LoS condition, it ignores multipath contributions, because the ToF of the LoS signal corresponds to the first peak, appearing in the correlation sequence. The upper plot of Fig. 2, obtained from experimental data, shows a received signal as a function of time, obtained by transmitting a narrowband chirp, spanning the [38 kHz-42 kHz] interval in $200 \mathrm{~ms}$. Multipath components are present, spaced by a few $\mathrm{ms}$ from each other, and overlapping with the LoS contribution. The lower plot shows the correlation between the received signal and a previously stored template. Distinct peaks can be observed in the correlation sequence. Most of them can be attributed to multipath contributions. Moreover, since the first peak corresponds to the LoS path, its abscissa corresponds to the ToF, and multipath effects can easily be discriminated and ignored. Notice that a single pulse is transmitted, rather than a continuous pulse train. Thus, the system operational range is not limited by a pulse retransmission period. Also, the chirp duration may be increased to improve the $T o F$ measurement accuracy at a price of an increased measurement duration. In fact, an increased pulse duration results in a longer integration time and in an increased noise rejection when the correlation sequence is evaluated.
In a real scenario, range limiting factors are the sensitivity of the acoustoelectric transducers, i.e. the minimum detectable signal level, the $S N R$, that can affect the correlation algorithm after a chirp pulse has been acquired, and the memory depth $N_{A}$ of the receiver's Data Acquisition System (DAQ). In fact, since the beacon starts listening when the mobile node transmits a pulse, the maximum measurable $T o F$ is upper bounded by $N_{A}$ times the DAQ sampling period minus the pulse duration. In a multichannel system, a single pulse may be transmitted and collected simultaneously by the beacons, or individual transmission may be carried out sequentially between the mobile node and each beacon. While the first approach reduces the measurement duration, the second is potentially safer, avoiding cross-talk among beacons' channels.

The signal propagation has been modeled as in [28], assuming that the propagation channel is not frequency selective. This model assumes that a sinusoidal acoustic wave is transmitted in air with a sound pressure level $S P L_{t x}$, expressed in $\mathrm{dB}$, and that the air is characterized by an absorption coefficient $\alpha$, expressed in $\mathrm{dB} / \mathrm{cm}$, obtaining the received sound pressure level $S P L_{r x}$ as

$$
S P L_{r x}=S P L_{t x}-20 \log \left(\frac{d}{30}\right)-\alpha d,
$$

where $d$ is the distance between the transmitter and the receiver, expressed in $\mathrm{cm}$. By assuming that the receiving transducer has a flat frequency response and converts the received acoustic wave into an electrical signal with rms voltage $V_{r m s}$, with a sensitivity $S_{x}$, expressed in $\mathrm{V} / \mu \mathrm{Pa}$, we have

$$
V_{r m s}=p_{r e f} 10^{S P L_{r x}+S_{x, d B}}, S_{x, d B}=20 \log \left(\frac{S_{x}}{10}\right)
$$

where $p_{\text {ref }}$ is a reference level, assumed equal to $20 \mu \mathrm{Pa}$, and $S_{x, d B}$ is the sensitivity of the acoustoelectric transducer, expressed in $\mathrm{dB}$.

Furthermore, the propagation velocity $v$ of the sound wave has been modeled, as a function of the temperature, as [29]

$$
v=v_{0} \sqrt{1+\frac{T}{273}},
$$

where $T$ is the ambient temperature, expressed in Celsius degrees, and $v_{0}=331.4 \mathrm{~m} / \mathrm{s}$ is the speed of sound in air at the reference temperature $T_{0}=273.16 \mathrm{~K}$. Equations (4) to (6) describe the $T o F$ and the received amplitude of a sinewave, transmitted at a distance $d$, and may also be applied to narrowband chirp signals.

\section{SiMULATION AND MEASUREMENT RESULTS}

The proposed system architecture was characterized by means of Montecarlo simulations, considering an expanded uncertainty of $1 \mathrm{~cm}$ as targeted positioning accuracy. Subsection A describes the results of set of simulations, aimed at investigating system level design criteria, considering parameters such as the size of the beacon panel, the number of beacons, and the ToF measurement jitter. The effects of such parameters on ranging and positioning accuracy were considered. Another set of simulations, described in subsection 


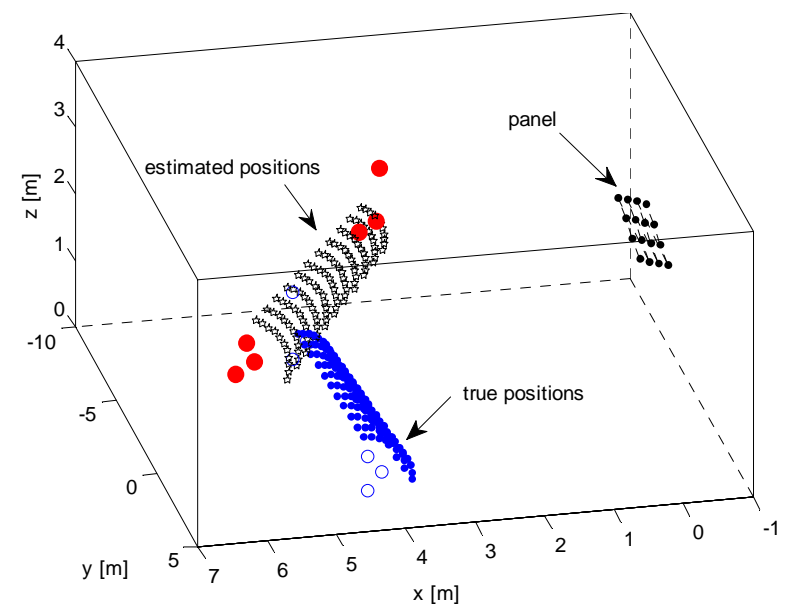

Fig. 3: beacon panel, mobile node true positions (points), and fixed anchors (circles). The stars show the estimated positions before applying the compensation procedure. Filled circles mark the apparent positions of the fixed anchors.

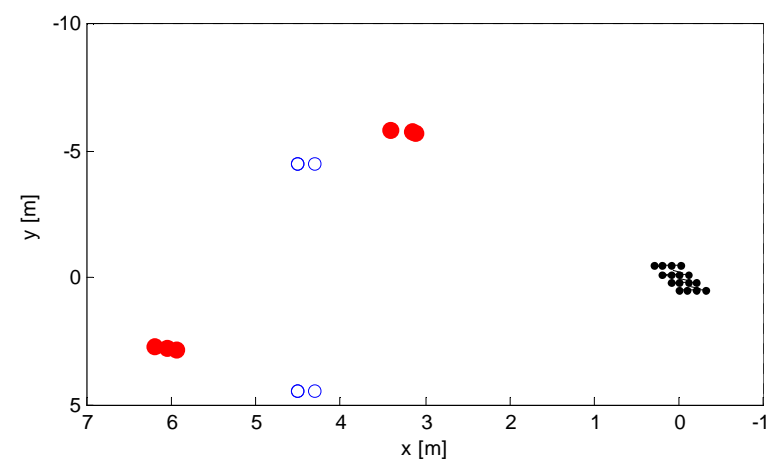

Fig. 3b: projection on the $x-y$ plane of the coordinates of the beacon panel (points on the right), of the true anchors' positions (circles), and on the apparent anchors' positions (filled circles).

$B$, investigated the chirp transmission, with the aim of assessing the signal characteristics ensuring a given ToF accuracy. Experimental results, validating the ranging performance, are shown in subsection $\mathrm{C}$.

\section{A. System level performance analysis}

The considered positioning system, shown in Fig. 3, features a square $4 \times 4$ beacon grid, placed on a square panel with a size of $1 \mathrm{~m}$. The considered artifact is a portion of a conical surface, the radius decreasing through its length, placed at about $5 \mathrm{~m}$ off the panel, represented by a point matrix in Fig. 3. Two sets of 3 fixed anchors each were also considered, described by circles, placed at two opposite sides of the artifact. Each $T o F$ measurement was simulated by a Gaussian random variable (r.v.). The r.v.'s mean value was obtained from the distance between the involved nodes, converting it into a propagation time using (6). Throughout the simulations, a temperature of $23{ }^{\circ} \mathrm{C}$ has been assumed and kept into account. For a targeted accuracy of $1 \mathrm{~cm}$, temperature control is needed, because (6) shows that a temperature variation of 1 ${ }^{\circ} \mathrm{C}$ can cause a systematic ranging error of about $0.18 \mathrm{~cm}$ per meter and per degree Celsius, corresponding to an error of about $1 \mathrm{~cm}$ at a distance of $5 \mathrm{~m}$. Moreover, the standard

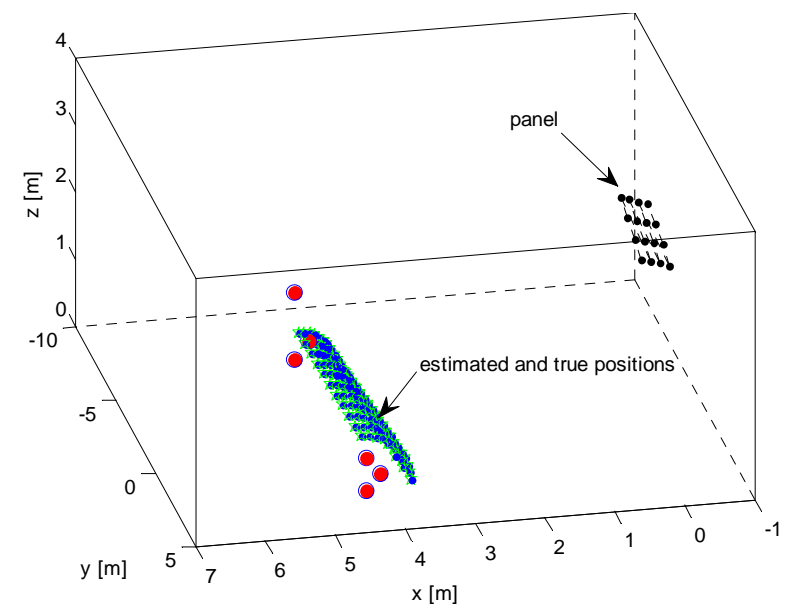

Fig. 4: position estimation after applying the compensation procedure. The true positions (points) and the estimated ones (stars) nearly coincide. Filled circles mark the apparent positions of the fixed anchors after applying the correction, nearly coinciding with the true positions (circles).

Table I: effect of repeated position measurements

\begin{tabular}{|c|c|c|c|c|}
\hline$M_{\text {cal }}$ & $M$ & $e_{\max }$ & $e_{\text {mean }}$ & $e_{\text {std }}$ \\
\hline 100 & 1 & $5.2 \mathrm{~mm}$ & $1.6 \mathrm{~mm}$ & $1 \mathrm{~mm}$ \\
\hline 100 & 10 & $1.9 \mathrm{~mm}$ & $0.53 \mathrm{~mm}$ & $0.32 \mathrm{~mm}$ \\
\hline 100 & 100 & $0.6 \mathrm{~mm}$ & $0.2 \mathrm{~mm}$ & $0.1 \mathrm{~mm}$ \\
\hline
\end{tabular}

Table II: effect of variable number of beacons

\begin{tabular}{|c|c|c|c|}
\hline$N \times N$ & $e_{\max }$ & $e_{\text {mean }}$ & $e_{\text {std }}$ \\
\hline $2 \times 2$ & $8.1 \mathrm{~mm}$ & $3.4 \mathrm{~mm}$ & $1.5 \mathrm{~mm}$ \\
\hline $3 \times 3$ & $7.7 \mathrm{~mm}$ & $3.1 \mathrm{~mm}$ & $1.3 \mathrm{~mm}$ \\
\hline $4 \times 4$ & $7.4 \mathrm{~mm}$ & $2.2 \mathrm{~mm}$ & $1.2 \mathrm{~mm}$ \\
\hline $5 \times 5$ & $6.6 \mathrm{~mm}$ & $1.2 \mathrm{~mm}$ & $3.1 \mathrm{~mm}$ \\
\hline $6 \times 6$ & $4.8 \mathrm{~mm}$ & $1.5 \mathrm{~mm}$ & $0.8 \mathrm{~mm}$ \\
\hline
\end{tabular}

deviation $\sigma_{T o F}$ of the Gaussian r.v. was used to model ToF measurement jitter.

In order to test the effectiveness of the proposed procedure, an orientation error was assumed in placing the beacon panel, equal to $0.1 \pi$ radians both in the azimuth angle and in the elevation angle. A $\sigma_{T o F}$ of $1 \mu$ s was initially assumed. As expected, prior to estimating and applying the transformation $R$, the estimated positions of the mobile node, also shown in Fig. 3, are affected by a large error.

After estimating $R$ using the apparent positions of the fixed anchors (filled circles in Fig. 3 and $3 b$ ) and the known positions (circles in Fig. 3 and 3b), the error is greatly reduced, leading to Fig. 4. Here, the corrected position estimates are nearly superimposed to the true positions. Under the stated conditions, the maximum positioning error $e_{\max }$, defined as the distance between the estimated position and the true ones, is approximately $0.5 \mathrm{~cm}$.

The system performance was analyzed by means of four sets of Montecarlo simulations, with the aim of assessing the effect of repeated measurements, variable number of transceivers in the beacon panel, panel size, and jitter level. 
Table III: effect of variable $T o F$ jitter $\sigma_{T o F}$

\begin{tabular}{|c|c|c|c|}
\hline$\sigma_{\text {ToF }}$ & $e_{\max }$ & $e_{\text {mean }}$ & $e_{\text {std }}$ \\
\hline $50 \mu \mathrm{s}$ & $316 \mathrm{~mm}$ & $144 \mathrm{~mm}$ & $62 \mathrm{~mm}$ \\
\hline $10-\mu \mathrm{s}$ & $55 \mathrm{~mm}$ & $19 \mathrm{~mm}$ & $9 \mathrm{~mm}$ \\
\hline $5 \mu \mathrm{s}$ & $32 \mathrm{~mm}$ & $9.1 \mathrm{~mm}$ & $5.9 \mathrm{~mm}$ \\
\hline $1 \mu \mathrm{s}$ & $7.4 \mathrm{~mm}$ & $2.2 \mathrm{~mm}$ & $1.2 \mathrm{~mm}$ \\
\hline
\end{tabular}

The first set of simulations was carried out, assuming to perform repeated measurements, followed by averaging, for each position assumed by the mobile node, leading to the results shown in Table I. In this scenario, the calibration, performed only when the portable beacon panel is moved, is obtained by averaging $M_{c a l}=100$ measurements, while the estimation of the mobile node position is obtained by averaging $M$ consecutive measurements. Moreover, the ToF jitter $\sigma_{T o F}$ was assumed equal to $1 \mu$ s. Table I shows the maximum value $e_{\text {max }}$, the mean value $e_{\text {mean }}$, and the standard deviation $e_{s t d}$ of the positioning error, evaluated across the set of positions assumed by the mobile node. It can be observed that the performance improves with $M$, seemingly by a factor $\sqrt{M}$.

The second set of simulations was carried out by assuming single measurements, but with different numbers of transceivers in the square beacon panel with a $1 \times 1 \mathrm{~m}^{2}$ size, leading to the results shown in Table II. This time, $M=M_{c a l}=1$ was assumed. Also in this case, it can be observed that increasing the number of transceivers improves the positioning accuracy. As expected, the $3^{\text {rd }}$ line of Table II shows a worse performance than that reported in the $1^{\text {st }}$ line of Tab. I, because the usage of repeated measurements in the calibration phase leads to a better calibration accuracy.

The third set of simulations was carried out by assuming a variable panel size $L$, again with $M=M_{c a l}=1$. The analysis was repeated by assuming a $2 \times 2$ grid, $3 \times 3$ grid, a $4 \times 4$ grid, and a $5 \times 5$ grid, obtaining the curves shown in Fig. 5, that show the maximum positioning error $e_{\max }$ as a function of $L$. For values of $L$ below $30 \mathrm{~cm}$, reducing $L$ leads to a large positioning error, regardless of the number of beacons. Conversely, when $L>60 \mathrm{~cm}$, increasing $L$ introduces a moderate performance improvement. Moreover, it can be observed that the curves obtained for grids with increasing numbers of nodes are very similar to each other. This shows that the panel size is a more critical factor than the numbers of beacons embedded in the panel. In fact, when $L$ is small, the panel beacons are very close to each other and their ToF measurements bring very similar information, such that the triangulation algorithm is required to solve an ill-conditioned problem. Fig. 5 also shows that $L=1 \mathrm{~m}$ is approximately the minimum value of $L$ corresponding to a maximum positioning error of $1 \mathrm{~cm}$ or less for a minimum complexity $2 \times 2$ grid. Such value of $L$ was considered when designing the experimental setup, since it corresponds to the design targeted uncertainty.

When running the simulations, the estimated parameters of the transformation $R$ were stored, evaluating their distribution for different values of $L$. The estimators were compared to the reference value, that is the parameters of $\mathrm{R}$ calculated by assuming noiseless $T o F$ measurements. For each parameter, all

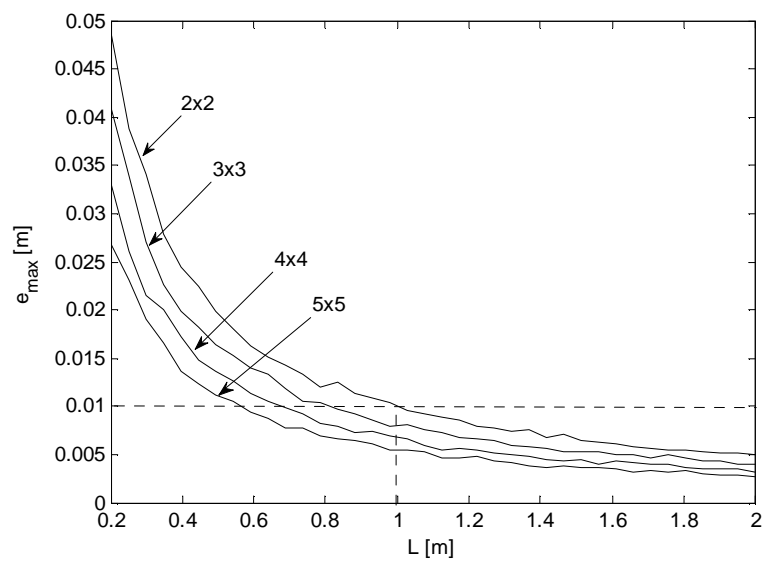

Fig. 5: maximum positioning error, as a function of the size $L$ of a square beacon panel, for various numerosities of the beacon matrix.

the estimators appear to be Gaussian distributed and unbiased. Moreover their standard deviation behaves like the $e_{\max }$ curves of Fig. 5.

The last set of simulations was carried out by changing the jitter level, leading to the results shown in Table III, obtained by considering a $4 \times 4$ beacon grid, and $M=M_{c a l}=1$. Simulations show that the $T o F$ jitter has a great influence on the achievable performance. In fact, the mean value, the maximum value, and the standard deviation of the positioning error seemingly scale linearly with $\sigma_{T o F}$.

\section{B. Chirp transmission performance analysis}

In order to analyze the achievable ToF accuracy when chirp transmissions are used, Montecarlo simulations were carried out. We used the models (3) to (6), and assumed the typical parameters of a Murata MA40S4S piezoelectric transducer, that is a maximum output sound pressure level $S P L_{t x}=120 \mathrm{~dB}$, and a sensitivity $S_{x, d B}=-63 \mathrm{~dB}$ [30]. Such a device has been used in the experimental verifications, described in subsections III.C and IV.

The simulation environment was completed by assuming a uniform quantization of the received signal, and that the receiver is affected by an Additive White Gaussian Noise (AWGN).

Then, a ranging scenario was considered: the distance between two transceivers was considered varying in the interval $[1 \mathrm{~m}, 5 \mathrm{~m}]$, and an AWGN at the output of the receiving transducer was assumed, with a standard deviation of $1 \mathrm{mV}$. The contribution of quantization to $T o F$ error was made negligible, by assuming an ideal Automatic Gain Control and a resolution of 10 bit in the quantizer. As a first step, Signal to Noise Ratio $(S N R)$ at the receiver's output and the $T o F$ distribution were investigated, assuming a chirp pulse spanning the $[38 \mathrm{kHz}-42 \mathrm{kHz}]$ interval in $2 \mathrm{~ms}$. Thus, Fig. 6 shows the maximum $S N R$, in $\mathrm{dB}$, as a function of the distance between the transceivers. As anticipated, by assuming that the chirp pulse is transmitted both in air, and also via an electric channel, the $T o F$ can be estimated by correlating the two received replicas of the chirp, and finding the time corresponding to the first correlation peak. The estimation error in this case is upper bounded by half of the sampling period $T_{S}$, with $T_{S}=5 \mu \mathrm{s}$ in the 


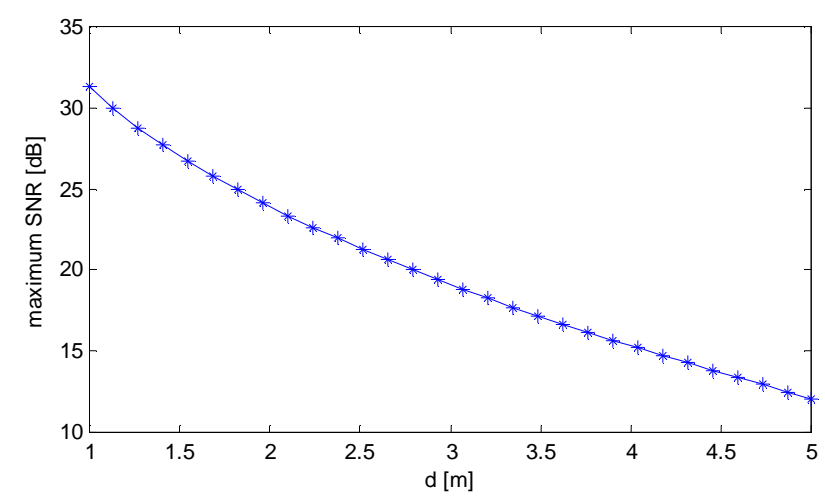

Fig. 6: $S N R$, evaluated during the chirp transmission as a function of distance between two ultrasonic transceivers, at the receiver output.

considered simulations, and is dominated by bias that changes with ToF according to a saw-tooth law. Notice that the correlation sequence presents a set of peaks, adjacent to the one corresponding to the $T o F$, spaced by the reciprocal of the chirp center frequency. In presence of channel and system noise, an adjacent peak may be erroneously selected, leading to a large ToF estimation error. For instance, for the considered chirp pulse with a center frequency of $40 \mathrm{kHz}$, the erroneous peak selection would correspond to a timing error of $25 \mu \mathrm{s}$. Such a phenomenon, also known as narrowband ambiguity, is inherent to the usage of narrowband chirp signals [34]. The occurrence of narrowband ambiguity errors depends on the $S N R$ at the receiver's input and on the chirp duration. For the considered chirp, the probability of experiencing such phenomenon is negligible for an $S N R$ of $5 \mathrm{~dB}$ or higher.

In order to improve the achievable accuracy, interpolation techniques have been applied, as in [31], fitting the correlation sequence in the proximity of a peak to a parabolic law and finding its maximum. The proposed procedure defines a new ToF estimator, whose root mean square error ( $r m s e)$ is plotted in Fig. 7, together with the rmse obtained in absence of interpolation. Since the rmse is reduced by almost one order of magnitude, the interpolation effectively refines the $T o F$ estimator obtained by simple peak identification. Moreover, the residual error appears Gaussian distributed in the considered distance range, with a small residual bias.

\section{Experimental results}

The achievable ranging accuracy was validated experimentally using the setup depicted in Fig. 8 with the aim of assessing the ranging sensitivity. Two Murata MA40S4S transducers were used [30], with a stated $3 \mathrm{~dB}$ directivity of $80^{\circ}$.

In particular, a linear chirp $s(t)$, spanning the $[38 \mathrm{kHz}-$ $42 \mathrm{kHz}$ ] interval in $2 \mathrm{~ms}$, was generated using an Agilent 33220A function generator. The received signals were acquired by an Agilent 54261A Digital Oscilloscope, configured to store records of 2000 samples at a sampling rate of $400 \mathrm{ksample} / \mathrm{s}$, with a resolution of 8 bits. Channel 1 of the DSO acquires the signal $s_{a}(t)$, available at the output of the receiving transducer, while Channel 2 collects the chirp signal $s_{e}(t)$, received through

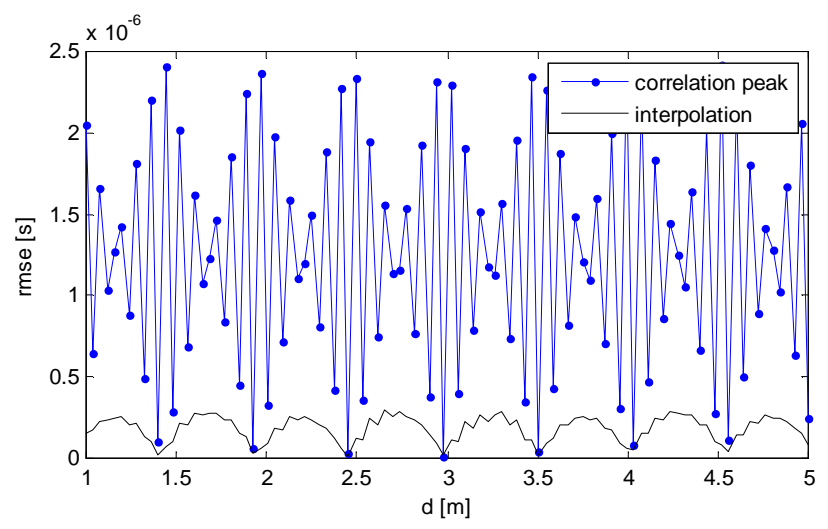

Fig. 7: root mean square error (rmse) of the considered $T o F$ estimators, based on chirp correlation (blue) and chirp correlation followed by data interpolation in a neighborhood of a correlation peak.

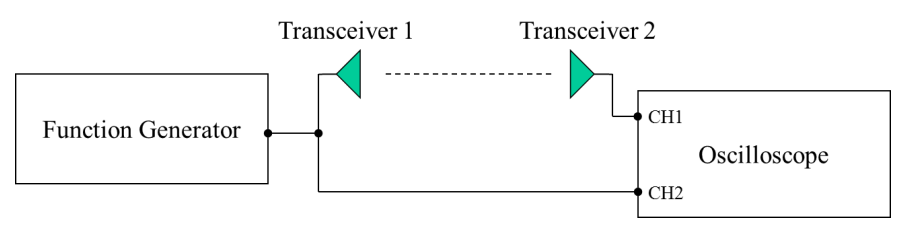

Fig. 8: experimental setup.

Table IV: effects of various parameters on system performance

\begin{tabular}{|c|c|}
\hline Parameter & Effect on performance \\
\hline Number of measurements $M$ & Accuracy improves as $\sqrt{M}$ \\
\hline ToF jitter $\sigma_{T o F}$ & $\begin{array}{c}\text { Accuracy improves linearly with } \\
\text { decreasing } \sigma_{T o F}\end{array}$ \\
\hline $\begin{array}{c}\text { Number of transceivers (fixed } \\
\text { panel size) }\end{array}$ & $\begin{array}{c}\text { Small accuracy improvements } \\
\text { when increased }\end{array}$ \\
\hline $\begin{array}{c}\text { Panel size (fixed number of } \\
\text { transceivers) }\end{array}$ & $\begin{array}{c}\text { Minimum size of } 1 \text { m between } \\
\text { each transceiver required for a } \\
\text { 2x2 grid. Larger spacing } \\
\text { introduces small accuracy } \\
\text { improvements }\end{array}$ \\
\hline $\begin{array}{c}\text { Chirp detection without } \\
\text { interpolation }\end{array}$ & $\begin{array}{c}\text { ToF maximum error and jitter } \\
\text { dominated by the sampling period }\end{array}$ \\
\hline Thirp detection with interpolation & $\begin{array}{c}\text { ToFimum error and jitter } \\
\text { much lower than the sampling } \\
\text { period, requires additional } \\
\text { processing }\end{array}$ \\
\hline Chirp duration & $\begin{array}{c}\text { Improved accuracy, at a price of } \\
\text { increased measurement duration }\end{array}$ \\
\hline Cross talk between receiving \\
beacons & $\begin{array}{c}\text { Prevented by sequentially } \\
\text { transmitting chirp pulses to } \\
\text { individually enabled beacons }\end{array}$ \\
\hline
\end{tabular}

the electrical channel. The two Murata piezoelectric transducers were initially placed at a distance of approximately $169 \mathrm{~cm}$, and a train of chirp pulses was transmitted, leading to the results in Fig. 9, that show both signals, simultaneously acquired, as a function of time. Notice that the envelope of the output of the Murata transducer is not constant, because its frequency response is not flat. Under the experimental conditions, the correlation sequence of the two collected signals, shown in Fig. 10, has a peak corresponding to the sample 1646. Then, the distance between the transceivers was increased by $1 \mathrm{~cm}$, and the measurements were repeated, 

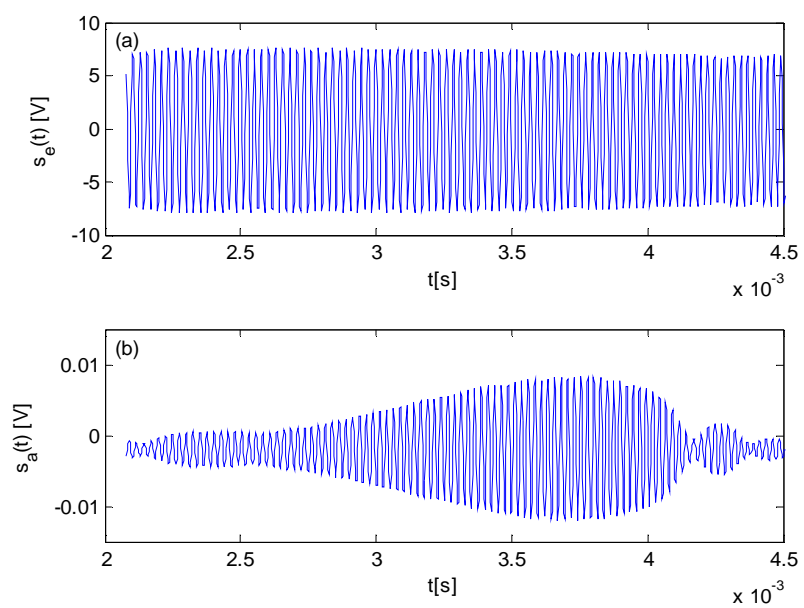

Fig. 9: chirp pulse, received through an electric channel (a) and through the acoustic channel, collected at the transducer's output (b).

obtaining a peak in the correlations sequence corresponding to the sample 1658. Since the acquisition rate is $400 \mathrm{ksample/s}$, the shift of 12 samples corresponds to a time shift of $30 \mu \mathrm{s}$. By recalling that the speed of sound is approximately $343 \mathrm{~m} / \mathrm{s}$, this corresponds to an estimated distance variation of $30 \mu \mathrm{s} \cdot 343$ $\mathrm{m} / \mathrm{s} \cong 1.03 \mathrm{~cm}$. Thus, the setup sensitivity is fairly consistent with the expected one.

Table IV summarizes the results obtained in subsections A, $\mathrm{B}$, and $\mathrm{C}$, that may be useful to design a working system and correctly exploit the available degrees of freedom. Simulation results show that, in order to meet an accuracy of $1 \mathrm{~cm}$ or better, ToF jitter is the most critical parameter, and that repeated measurements, interpolation, and averaging can be profitably used to reduce any Type A contribution to the measurement uncertainty [31][32].

Following such results, a more developed system was deployed, aimed at achieving a better ranging accuracy. In particular, the chirp signal was collected using an Agilent U2331A Data Acquisition System, capable of collecting signal with a sampling rate of up to $1 \mathrm{Msample/s.} \mathrm{In} \mathrm{the} \mathrm{considered}$ setup, a control unit sends a single chirp pulse to the mobile node electroacoustic transducer using a wire, and simultaneously sends a trigger to the beacon. The beacon starts listening for incoming signals, and collects the ultrasonic pulse. Then, the ToF is estimated using correlation between the signal received at the output of the beacon's acoustoelectric transducers and a locally stored signal replica, assumed to be started by the trigger. In a range of a few meters, the electric signal propagation through a cable can be considered instantaneous with respect to the acoustic one. Thus, the receiver nodes can infer the $T o F$ by estimating the correlation between the two incoming signals. Moreover, rather than a synthetic chirp pulse, a template was acquired at a distance of $1 \mathrm{~m}$ as suggested in [33], collecting 500 records and performing a record average to reduce noise effects on the template itself. Such a solution better keeps into account modifications induced by the propagation medium on the transmitted signal [33]. This method also compensates for any latency introduced by the electronic circuitry or by the signal processing, that

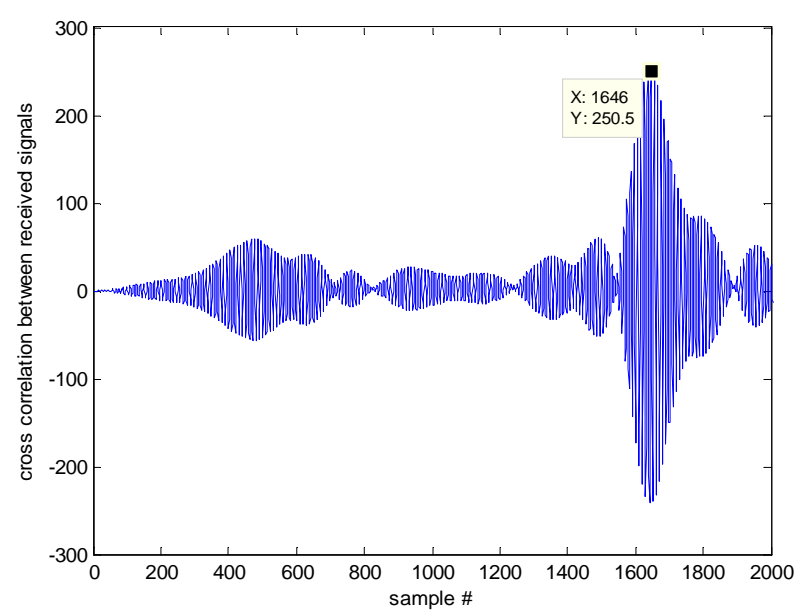

Fig. 10: correlation between the received signals (zoomed in)

equally affect both the template and the signal collected during the measurement phase. Using the presented setup, range estimations were performed by putting the two obtained transceivers at distances of $1.002 \mathrm{~m}, 1.182 \mathrm{~m}, 1.783 \mathrm{~m}$, and $1.882 \mathrm{~m}$, using a leveling rod. For each considered distance between the transmitter and the receiver, 500 consecutive chirp pulses were transmitted and collected. Then, correlation analysis was carried out between the collected signal and the template to assess the chirp $T o F$, subsequently averaging the results. Under such conditions, the obtained mean error equaled approximately $0.9 \mathrm{~mm}$, as shown in Fig. 11, proving the good potential of the technique.

Finally, tests were repeated by applying an azimuthal rotation to one of the transceivers, in order to characterize the ranging accuracy when the transceivers do not face each other. Experimental results suggest that an azimuthal rotation of less than $45^{\circ}$ does not introduce a significant performance degradation. A perceivable accuracy degradation has been observed for a rotation of $90^{\circ}$, consistent with the directivity of the adopted transceivers. Under such conditions, the significant reduction of the received signal level introduces a large uncertainty on the zero crossing of the received chirp signal, worsening the performance of the correlation algorithm.

\section{POSITIONING SYSTEM}

Following the consolidation of the ranging system, a positioning system was developed, whose characteristics are summarized in Table V. The beacon panel was realized by placing 7 beacons in an hexagonal configuration, as shown in Fig. 12. The experimental setup is depicted in fig. 13, where the blue circles on the right mark the 7 beacons, mounted on a rigid panel, while the red circle on the left marks a plane region, where the mobile node was placed. A longer chirp was used, with a duration $t_{d}=200 \mathrm{~ms}$, so as to improve the accuracy of the ToF measurement. Since the usage of anchors to compensate for panel placement errors was validated extensively via simulations (see section III), and since in the lab environment the beacon panel can be placed with good accuracy, the activities were aimed at assessing the system 

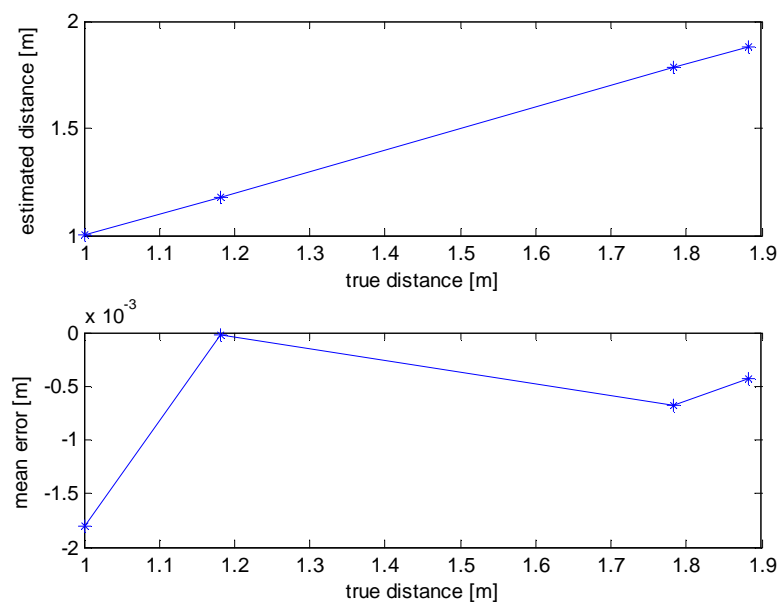

Fig. 11: experimental results. Ranging accuracy obtained using a Data Acquisition System, 500 acquisitions, and an experimentally collected template.

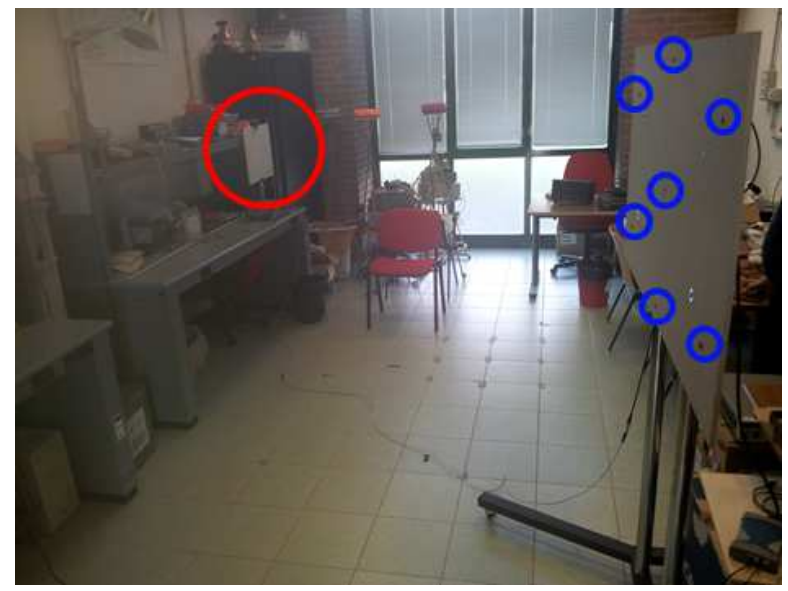

Fig. 13: experimental setup. The circles on the right mark the panel mounted beacons, while the large circle on the left marks a planar panel where the mobile node was placed for testing purposes.

accuracy in measuring the relative position of a mobile node, referred to the beacon panel.

The measurement is carried out by first estimating the distance between the mobile node and each beacon, as described in subsection III.C. Then, the system estimates the position by using the least squares method to fit the distance between the mobile node and each of the beacons to the measured distances.

The experimental setup shown in Fig. 13 models a disadvantageous situation, since each beacon experiences an individual azimuthal rotation with respect to the mobile node, close to $80^{\circ}$. Such value corresponds to the $3 \mathrm{~dB}$ directivity of the adopted transducers, and may correspond to strong attenuation of the received signals. The setup was chosen to highlight any influence of transceivers' directivity on the ToF measurements.

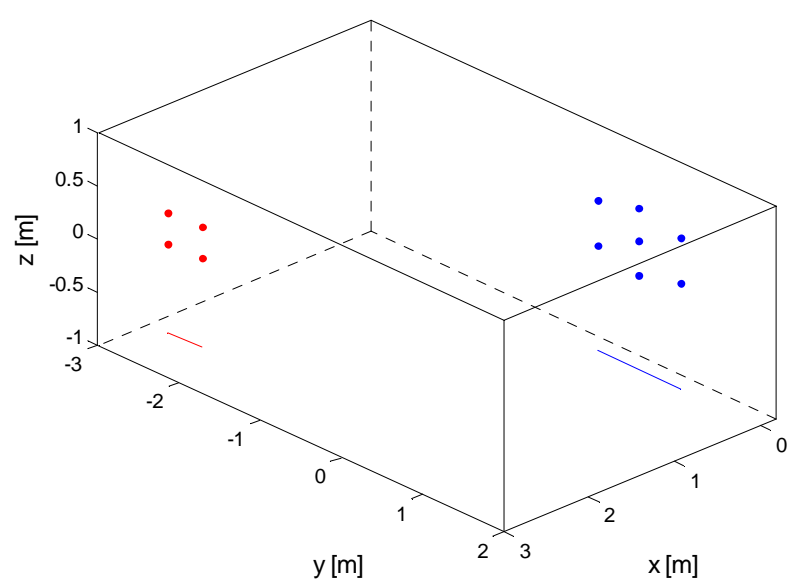

Fig. 12: measurement results. The points on the right correspond to the 7 panel beacons, placed in the vertexes and in the center of an hexagon (see circles on the right in Fig. 13). The points on the left mark the mobile node estimated positions (see large circle on the left in Fig. 13). Solid lines are the projections of the various positions on the floor.

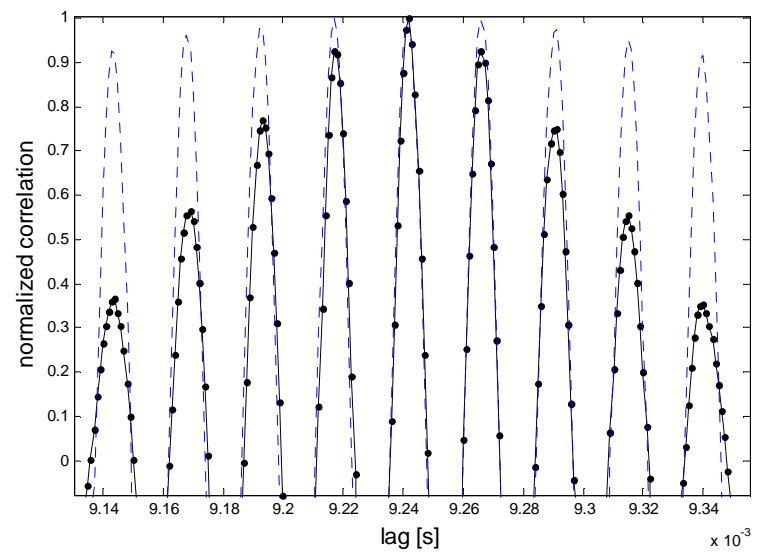

Fig. 14: correlation peaks between a stored template and the received chirp (dotted curve). The solid curve is obtained by feeding the chirp signals to a hard limiter, prior to performing the cross correlation.

Table V: characteristics of the positioning system

\begin{tabular}{|c|c|c|c|c|}
\hline $\begin{array}{c}\text { Number } \\
\text { of } \\
\text { beacons }\end{array}$ & $\begin{array}{c}\text { Beacon } \\
\text { configuration }\end{array}$ & $\begin{array}{c}\text { Panel } \\
\text { size }[\mathrm{m}]\end{array}$ & $\begin{array}{c}\text { Chirp } \\
\text { duration }\end{array}$ & Chirp detection \\
\hline 7 & Hexagonal & $1.2 \times 1$ & $200 \mathrm{~ms}$ & $\begin{array}{c}\text { Uses interpolation } \\
\text { and hard limiting }\end{array}$ \\
\hline
\end{tabular}

For each beacon, an individual calibration was preliminarily carried out, collecting a specific template. Each time, the mobile node was aligned to the beacon and placed at a distance $d_{T}=0.940 \mathrm{~m}$, known with an extended uncertainty of less than $1 \mathrm{~mm}$, measuring at the same time the temperature $T_{T}$ and the relative humidity $h_{T}$. Such an approach effectively compensates specific latencies of each beacon-mobile node channel. Moreover, during the position measurement, the temperature $T_{M}$ and the humidity $h_{M}$ were measured, and, for each beacon, their effect on the distance estimation $\hat{d}$ was compensated, using the following 


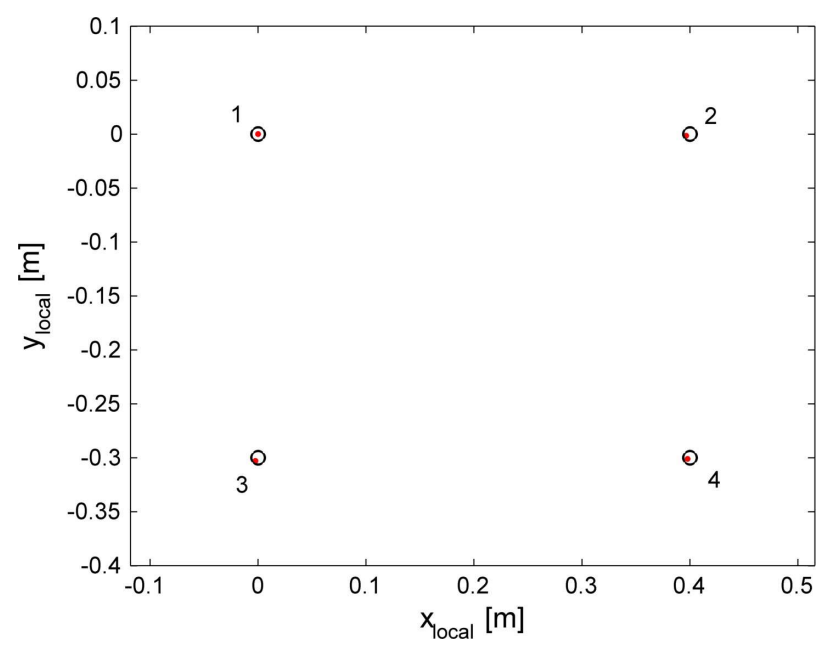

Fig. 15: experimental results, four positions assumed by the mobile node (circlets) on the testing planar surface and the corresponding estimated positions (dots). The points are shown in a local reference frame, the origin being the upper left position assumed by the mobile node.

$$
\begin{aligned}
& \hat{d}=\left(\hat{t}_{T o F}+\frac{d_{T}}{v_{T}}\right) v_{M}, \\
& v_{T}=v_{0} f\left(h_{T}, T_{T}\right) \sqrt{1+\frac{T_{T}}{273}}, \\
& v_{M}=v_{0} f\left(h_{M}, T_{M}\right) \sqrt{1+\frac{T_{M}}{273}},
\end{aligned}
$$

derived by extending (6), where $\hat{t}_{T o F}$ is the estimated $T o F, v_{T}$ is the speed of sound in air during the acquisition of the template, $v_{M}$ is the speed of sound during the position measurement, and $f(\cdot, \cdot)$ is a corrective factor, given by [33]

$$
f(h, T)=h\left(1.0059 \cdot 10^{-3}+1.7777 \cdot 10^{-7}(T+17.78)^{3}\right) \text {, }
$$

modeling the effect of relative humidity $h$ on the speed of sound in air when the absolute temperature equals $T$ [33]. Notice that (7)-(10) apply also when the template chirp and the one transmitted during the measurement are taken at different times, under different environmental conditions.

Moreover, in order to reduce measurement time and improve accuracy, the bipolar hard-limiter law

$$
y=\left\{\begin{array}{ll}
1, & x \geq 0 \\
-1, & x<0
\end{array},\right.
$$

was applied to both the received chirp and each stored template, prior evaluating their cross-correlation sequence. As shown in Fig. 14, when the correlation between the received chirp and the stored template is evaluated, this technique reduces the amplitude of secondary peaks surrounding the highest one, corresponding to the true delay between the processed signals. This approach reduces the occurrence probability of narrowband ambiguity, described in subsection III-B.
Notice that, while the results presented in section III were obtained using repeated chirp transmissions and averaging techniques, processing the chirp signals with the hard limiter provides a good $T o F$ accuracy even if single measurements are performed.

The system was repeatedly tested, providing a satisfactory performance. In particular, Fig. 15 shows four position assumed by the mobile node, as circlets, and the estimated positions, as points, corresponding to the positions of Fig. 12. Under such conditions the distance between the mobile node and the beacons exceeds $4 \mathrm{~m}$, while ultrasound positioning systems typically operate up to $2 \mathrm{~m}$. For easiness of analysis, the positions are represented in a local reference frame, the origin being in the upper left measured position. The positioning accuracy was assessed, comparing the measured positions, relative to the first one, with respect to the true relative positions. Under such conditions, we obtained a maximum positioning error of about $3.9 \mathrm{~mm}$, a mean positioning error of about $3.4 \mathrm{~mm}$, and a standard deviation of $0.8 \mathrm{~mm}$. The position measurement, carried out by sequentially polling the 7 beacons, completing the correlation and interpolation analysis, and triangulating the mobile node position, is completed in about $5 \mathrm{~s}$, where the acquisition lasted about $3 \mathrm{~s}$.

\section{CONCLUSIONS}

A technique for locating a mobile device, based on ultrasound signaling and Time of Flight measurements, has been presented and partially characterized. The approach, suitable for industrial applications, relies on a small set of fixed anchors and on a portable set of closely spaced beacons, that can be moved to guarantee Line of Sight transmission between the beacons and the mobile device to be located. The achievable performance was investigated as a function of various system parameters. Chirp signaling has been considered and analyzed as a mean of achieving low jitter Time of Flight measurements. Overall, a low cost positioning system was developed, based on ToF based range estimations, that measures the relative position of a mobile node with respect to the beacon set. The system has been successfully tested, meeting the targeted performance of sub-centimeter accuracy in a range up to $4 \mathrm{~m}$.

\section{REFERENCES}

[1] R. Mautz, "The challenges of indoor environments and specification on some alternative positioning systems," Proc. of 6th IEEE Workshop on Positioning, Navigation and Communication, WPNC 2009, pp. 29-36, DOI 10.1109/WPNC.2009.4907800.

[2] A. De Angelis, M. Dionigi, A. Moschitta, P. Carbone, "A Low-Cost Ultra-Wideband Indoor Ranging System," IEEE Transactions on Instrumentation and Measurement, Year 2009, Vol. 58, No. 12, pp. 3935 - 3942, DOI 10.1109/TIM.2009.2020834.

[3] A. Cazzorla, G. De Angelis, A. Moschitta, M. Dionigi, F. Alimenti, P. Carbone "A 5.6 GHz UWB Position Measurement System," IEEE Trans. Instr. Meas, Vol. 62, No. 3, March 2013, pp. 675-683.

[4] G. R. Olheft, "Electromagnetic field and material properties in ground penetrating radar," proc. 2nd Inter. Workshop on Advanced Ground Penetrating Radar, 2003, pp. 144-147. DOI: 10.1109/AGPR.2003.1207309.

[5] Lu Zhejun, Liu Wenyan, Huang Xiaotao, "Simulation and Performance Analysis for a Distributed Multi-station UWB Positioning System," Proc. of 2012 Second International Conference on Intelligent System 
Design and Engineering Application (ISDEA), Sanya, Hainan, China, January 6-7 2012, pp. 1486-1489, DOI 10.1109/ISdea.2012.526.

[6] Cemin Zhang, M.J. Kuhn, B.C. Merkl, A.E. Fathy, M.R. Mahfouz, "Real-Time Noncoherent UWB Positioning Radar With Millimeter Range Accuracy: Theory and Experiment," IEEE Transactions on Microwave Theory and Techniques, Vol. 58, No. 1, 2010 , pp. 9-20, DOI: 10.1109/TMTT.2009.2035945.

[7] Andreas Fink, Helmut Beikirch, "RSSI-based Indoor Localization using Antenna Diversity and Plausibility Filter," Proceedings of 6th IEEE Workshop on Positioning, Navigation and Communication 2009 WPNC'09.

[8] Y. Dama, R. Abd-Alhameed, F. Salazar-Quinonez, D. Zhou, S. Jones, S. Gao, "MIMO indoor propagation prediction using 3D shoot-and-bounce ray (SBR) tracing technique for $2.4 \mathrm{GHz}$ and $5 \mathrm{GHz}$," Proc. European Conference on Antennas and Propagation (EUCAP), Rome, Italy, Apr. 2011, pp. 1655 - 1658

[9] A. Moschitta, D. Macii, F. Trenti, S. Dalpez, A. Bozzoli, "Characterization of a geometrical wireless signal propagation model for indoor ranging techniques," Proc. of 2012 IEEE International Instrumentation and Measurement Technology Conference I2MTC, 2012, pp. 2598-2603, DOI 10.1109/I2MTC.2012.6229373.

[10] I. Neri, R. Centonze, M. L. Fravolini, A. Moschitta, "A simple ranging technique based on received signal strength measurements in a narrowband $2.4 \mathrm{GHz}$ channel: a space diversity approach," 2013 IEEE International Workshop on Measurement and Networking, Naples, Italy, October 7-8 2013.

[11] J. Blankenbach, A. Norrdine, "Position estimation using artificial generated magnetic fields," Proc. of 2010 IEEE International Conference on Indoor Positioning and Indoor Navigation IPIN 2010, pp. 1-5, DOI 10.1109/IPIN.2010.5646739.

[12] A.P. Sample, D.A. Meyer, J.R. Smith, "Analysis, Experimental Results, and Range Adaptation of Magnetically Coupled Resonators for Wireless Power Transfer," IEEE Transactions on Industrial Electronics, Vol. 58, No. 2, 2011, pp. 544-554, Digital Object Identifier: 10.1109/TIE.2010.2046002.

[13] Young-jae Ryoo, Jang-hyun Park, "Design and development of magnetic position sensor for magnetic guidance system of automated ground vehicle," Proceedings of 12th IEEE International Conference on Control, Automation and Systems (ICCAS 2012), pp. 988-991.

[14] Website of Polhemus. [online] http://www.polhemus.com.

[15] M. Dionigi, G. De Angelis, A. Moschitta, M. Mongiardo, P. Carbone, "A simple ranging system based on mutually coupled resonating circuits," Proc. of 2013 IEEE Instrumentation and Measurement Technology Conference I2MTC 2013, Minneapolis, MN, USA, May 69, 2013.

[16] Website of Bushnell, [online] http://www.bushnell.com.

[17] M. S. Rahmant, Md. Mejbaul Haquet, Ki-Doo Kim, "High Precision Indoor Positioning Using Lighting LED and Image Sensor," 14th International Conference on Computer and Infonnation Technology (ICCIT 2011) 22-24 December, 2011, Dhaka, Bangladesh.

[18] H. Yucel, T. Ozkir, R. Edizkan, A. Yazici, "Development of indoor positioning system with ultrasonic and infrared signals," Proc. of 2012 IEEE International Symposium on Innovations in Intelligent Systems and Applications INISTA 2012, pp. 1-4, DOI 10.1109/INISTA.2012.6246983.
[19] J.R. Gonzalez and C. J. Bleakely, "High-Precision Robust Broadband Ultrasonic Location and Orientation Estimation," IEEE Journal on Selected topics in Signal Processing, Vol. 3, no. 5, October 2009. DOI 10.1109/JSTSP.2009.2027795.

[20] D. Hauschildt, N. Kirchhof, "Improving indoor position estimation by combining active TDOA ultrasound and passive thermal infrared localization," IEEE 2011 8th Workshop on Positioning Navigation and Communication (WPNC), April 7-8 2011, Dresden, Germany, pp. 9499, DOI: 10.1109/WPNC.2011.5961022.

[21] N. B. Priyanta, A. Chakraborty, H. Balakrishnan, "The Cricket Location Support System," Proc. of $6^{\text {th }}$ ACM MOBICOM, Boston, MA, USA.

[22] Y. Fukuju, M. Minami, Hiroyuki Morikawa, T. Aoyama, "DOLPHIN: an autonomous indoor positioning system in ubiquitous computing environment," 2003 IEEE Workshop on Software Technologies for Future Embedded Systems, Japan, pp. 53-56, DOI 10.1109/WSTFES.2003.1201360.

[23] Kenji Nakahira, Tetsuji Kodama, Takeshi Furukashi, Hiroshi Maeda, "Design of Digital Polarity Correlators in a Multiple-User Sonar Ranging systems," IEEE Trans. on Instrumentation and Measurement, Vol. 54, No. 1, February 2005, DOI 10.1109/TIM.2004.839753.

[24] Yi Zhao, J.R. Smith, "A battery-free RFID-based indoor acoustic localization platform,", 2013 IEEE International Conference on RFID, pp.110-117, April 30-May 2 2013, doi: 10.1109/RFID.2013.6548143.

[25] C. Medina, J. C. Segura, A. De la Torre, "Ultrasound Indoor Positioning Based on a Low-Power Wireless Sensor Network Providing SubCentimeter Accuracy," Sensors 2013, 13, 3501-3526; doi:10.3390/s130303501.

[26] A. De Angelis, A. Moschitta, P. Carbone, M. Calderini, S. Neri, R. Borgna, M. Peppucci, "Design and characterization of an ultrasonic indoor positioning technique," Proc. of 2014 IEEE Instrumentation and Measurement Technology Conference I2MTC 2014, Montevideo, Uruguay, May $12-15,2014$.

[27] Pivato, P.; Dalpez, S.; Macii, D., "Performance evaluation of Chirp Spread Spectrum ranging for indoor embedded navigation systems," 2012 7th IEEE International Symposium on Industrial Embedded Systems (SIES), pp. 307-310, 20-22 June 2012.

[28] Arun T. Vemuri, "Using a fixed threshold in ultrasonic distance-ranging automotive applications," Texas Instruments Application Note SLYT481 [available online].

[29] H. W. When, P. R. Bélanger, "Ultrasound-Based Robot Position Estimation," IEEE Trans. on Robotics and Automation, Vol. 13, No. 5, October 1997, pp. 682-692, DOI 10.1109/70.631230.

[30] Murata MA40S4S datasheet, [online] www.murata.com.

[31] D. Marioli, C. Narduzzi, C. Offelli, D. Petri, E. Sardini, A. Taroni, "Digital Time-of-Flight Measurement for Ultrasonic Sensors," IEEE Trans. on Instrumentation and Measurement, Vol. 41, No. 1, Feb. 1992, DOI 10.1109/19.126639.

[32] Guide to the Expression of Uncertainty in Measurement (GUM), [online] www.bipm.org, as of 12/6/2014.

[33] L. Kleeman, R. Kuc, "Sonar Sensing," Chapter 21 of Springer Handbook of Robotics, edited by B. Siciliano and O. Khatib, pag. 491519, 2008, Springer, ISBN: 978-3-540-23957-4.

[34] A. Zeira, P.M. Schultheiss, "Realizable lower bounds for time delay estimation," , IEEE Transactions on Signal Processing, vol.41, no.11, pp. 3102-3113, Nov 1993. 